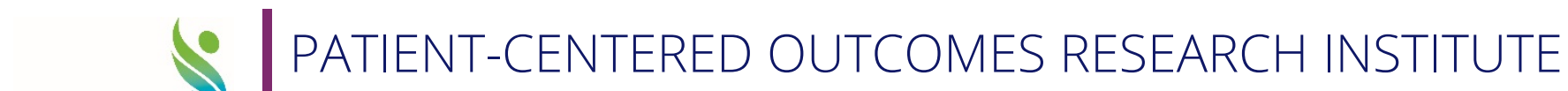 pcori ?. RESEARCH SUMMARY
}

\section{Linking Community-Based Organizations with Each Other, and with Hospitals and Health Clinics, to Help Connect Patients with the Services They Need}

Principal investigator

Albert Wu, MD, MPH
Organization

Johns Hopkins University

\section{What was the research about?}

Community-based organizations (CBOs) are nonprofit groups that work at the local level to improve life for people in need. CBOs can help people pay bills or find food, jobs, or places to live. CBOs can also help people with basic healthcare needs. Some doctors, hospital staff, or other healthcare professionals may not know about the services that $\mathrm{CBO}$ s offer to patients.

The research team wanted to learn if a program to link hospitals and clinics to CBOs could help keep patients in East and Southeast Baltimore, Maryland, from needing to go to the hospital or emergency room as often. The team compared patients who live near $\mathrm{CBOs}$ that were in this program with patients who live near CBOs that weren't in this program.

\section{What were the results?}

After a year, the research team found no differences between patients who live near CBOs that were in the program and patients who live near CBOs that weren't in the program. There were no differences in

- How often patients went to emergency rooms or hospitals

- How long patients stayed at hospitals if they were admitted

- How often patients at hospitals or clinics were told about the services of $\mathrm{CBO}$ s
- How often CBO clients were told about services at healthcare clinics

At the end of the program, staff members at inpatient and outpatient clinics in the study reported

- Fewer barriers referring their patients to CBOs

- More confidence in having the information they need about CBOs

- More patient referrals to the CBOs that were in the program than to the CBOs that weren't in the program

\section{Who was in the study?}

The study included 20 CBOs in low-income neighborhoods in East and Southeast Baltimore. The research team looked at healthcare records for 4,917 patients in the Johns Hopkins Health System. Patients lived in Baltimore and had many healthcare and service needs, such as trouble paying bills or finding a place to live.

\section{What did the research team do?}

The research team worked with $\mathrm{CBO}$ s and health professionals to create the program to improve how hospitals, clinics, and CBOs work together. The program included activities such as monthly meetings that involved all the CBOs in the program and meetings with Johns Hopkins Health System staff. 
The research team assigned the $\mathrm{CBOs}$, by chance, to one of two groups. One group was in the program; the other was not. Then, the research team looked at patient health records; the team grouped the patients' records by which CBO was closest to their homes. The research team looked at patients' health insurance billing information to see how many times patients went to an emergency room or hospital and how many days they stayed in the hospital if admitted. The research team compared patients who lived near $\mathrm{CBOs}$ that were in the program with patients who lived near CBOs that weren't in the program.

The research team also interviewed people who used the services of the CBOs. The team asked people about the services that CBOs offered and how many other CBOs the patients knew of. The team also asked about the problems patients faced in the previous month, such as finding a job. The research team surveyed staff members at Johns Hopkins Health System before and after the program. The surveys included questions about barriers that staff members faced when referring patients to CBOs. The surveys also asked whether staff members had the information they needed about the CBOs.

\section{What were the limits of the study?}

The research team didn't know if the patients visiting the hospital or clinics had ever been to their assigned CBOs, so the research team doesn't know if going to the $\mathrm{CBO}$ affected the study results. The number of CBOs in the study may have been too small to see if the program made a difference.

Future research could look at other ways to help teach patients about, and link patients to, the services in their communities.

\section{How can people use the results?}

Patients, doctors, and CBOs may be able to use the results from this study to work together to help people get the services they need to stay healthy. Teaching clinic staff members about CBOs may increase the number of patients the staff members refer to $\mathrm{CBO}$. However, making clinic staff aware of CBO services may not change how people use hospitals or emergency rooms.

To learn more about this project, visit pcori.org/Wu040. 